\title{
C-type Lectin Receptors: Signaling and Therapeutic Opportunities
}

\author{
J. Ruland ${ }^{1}$ \\ ${ }^{1}$ Institut für Klinische Chemie und Pathobiochemie, Klinikum \\ rechts der Isar, Technische Universität München
}

C-type lectin receptors (CLRs) that signal via the kinase Syk are an important class of pattern recognition receptors in the innate immune system. They recognize pathogen- and host-derived danger signals and drive several cellular responses, including phagocytosis, the production of reactive oxygen species (ROS), as well as altered gene expression. Best characterized is the role of CLR signaling in anti-fungal immunity, but accumulation evidence indicates additional essential functions for CLR activity in innate immunity against bacteria, viruses or parasites. Moreover, deregulated CLR signaling appears to contribute to inflammatory disease. Crucial for CLR-mediated inflammation and host defence are the engagement of NF-KB signaling pathway and inflammasome activation. NF-KB signaling by CLRs depends on the adapter protein Card9 that upon activation cooperates with Bcl10 and the paracaspase Malt1 to engage the canonical IKK pathway. This mechanism operates independent from CLR-induced ROS production and phagocytosis. Recent findings have indicated that PKCO is the critical kinase which directly phosphorylates Card9 at Thr231 and which subsequently triggers the signal-induced association of Card9 with Bcl10 and Malt 1 and the further recruitment of TAK1 for activation of the canonical NF-KB pathway. Moreover, Syk-mediated CLR signaling upon fungal recognition is crucial for IL- $1 \beta$ generation and is required for both proIL- $1 \beta$ synthesis and activation of the Nlrp3 inflammasome. While proIL-1ß synthesis selectively requires the Card9 pathway, inflammasome activation by fungi involves ROS production and potassium efflux. Thus, there is cross talk between Syk-coupled CLRs and Nlrp3, at least in the context of fungal infection. In this symposium I will discuss recent insights into the molecular regulation of CLR-mediated inflammatory effector function and its potential role in human disease together with opportunities to therapeutically manipulate these pathways.

\section{Conflict of Interest: None}

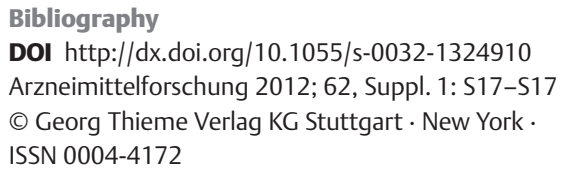

